Rhode Island College

Digital Commons @ RIC

2016

\title{
The Use of Probiotics in the Prevention of Clostridium Difficile Infection
}

Lindsay F. Mook

Rhode Island College

Follow this and additional works at: https://digitalcommons.ric.edu/etd

Part of the Nursing Commons

\section{Recommended Citation}

Mook, Lindsay F., "The Use of Probiotics in the Prevention of Clostridium Difficile Infection" (2016).

Master's Theses, Dissertations, Graduate Research and Major Papers Overview. 149.

https://digitalcommons.ric.edu/etd/149

This Major Paper is brought to you for free and open access by the Master's Theses, Dissertations, Graduate Research and Major Papers at Digital Commons @ RIC. It has been accepted for inclusion in Master's Theses, Dissertations, Graduate Research and Major Papers Overview by an authorized administrator of Digital Commons @ RIC. For more information, please contact digitalcommons@ric.edu. 



\title{
THE USE OF PROBIOTICS IN THE PREVENTION OF CLOSTRIDIUM DIFFICILE INFECTION
}

\author{
A Major Paper Presented
}

$$
\text { by }
$$

Lindsay F. Mook

Approved:

Committee Chairperson

Committee Members

(Date)

Committee Members

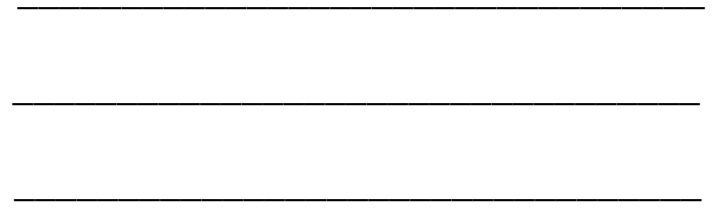

(Date)

(Date)

Director of Master's Program

$\longrightarrow$

(Date)

Dean, School of Nursing 
THE USE OF PROBIOTICS IN THE PREVENTION OF CLOSTRIDIUM DIFFICILE INFECTION

\author{
by \\ Lindsay F. Mook \\ A Major Paper Submitted in Partial Fulfillment \\ of the Requirements for the Degree of \\ Master of Science in Nursing \\ in \\ The School of Nursing \\ Rhode Island College \\ 2016
}




\begin{abstract}
Despite advances in the diagnosis and treatment of Clostridium difficile infection (CDI), the prevention of CDI, particularly in the inpatient hospital setting, remains a challenge. Clostridium difficile now rivals methicillin-resistant staphylococcus aureus (MRSA) as the most common pathogen to cause hospital acquired infections (HAI) in the United States. Hospitalized patients are considered to be especially high risk for CDI, and among inpatient cases, antibiotic treatment, especially with Fluoroquinolones has been an almost universal factor in the development of CDIs. One preventative measure that is incontinently used in the prevention of CDI is oral probiotics. Probiotic consumption is reported to exert a myriad of beneficial effects including enhanced immune response, balancing of colonic microbiota, treatment of diarrhea associated with travel and antibiotic therapy, control of rotavirus and clostridium difficile induced colitis. The American College of Gastroenterology recognizes the role of probiotics and included probiotics as a level $\mathrm{B}$ recommendation for the treatment of CDI. It has been hypothesized that the use of probiotics, as an adjunctive therapy in patients receiving antibiotics, may provide a key intervention in reducing primary CDI. The purpose of this study was to conduct a retrospective chart review to explore healthcare providers prescribing trends regarding Fluoroquinolone antibiotics and adjunctive probiotics in patients with hospital acquired CDI. The Synergy model was used to guide the study. Results indicated that probiotics are not frequently prescribed for hospitalized patients on Fluoroquinolones and when they are it is with inconsistency. Additional research is recommended to further assess the use of probiotics in conjunction with other classes of commonly used antibiotics; this study solely looked at Fluoroquinolones.
\end{abstract}





\section{Table of Contents}

Background/Statement of Problem .........................................

Literature Review........................................................ 11

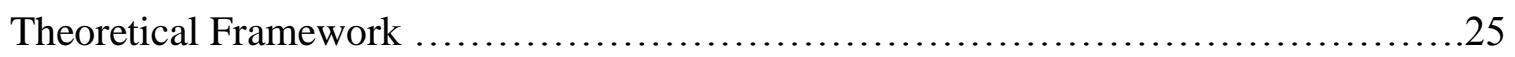

Method..................................................................27

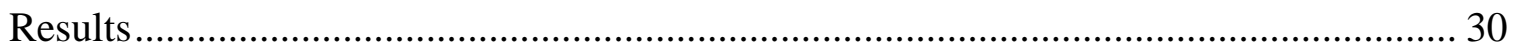

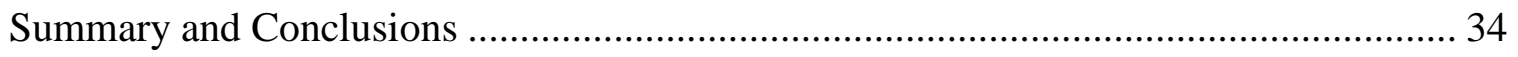

Recommendations and Implications for Advanced Nursing Practice .......................... 38

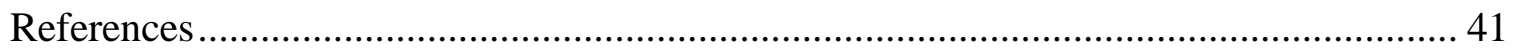

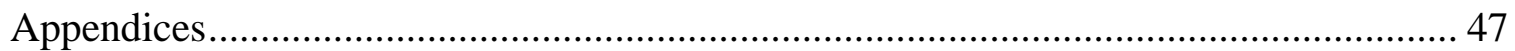




\section{THE USE OF PROBIOTICS IN THE PREVENTION OF CLOSTRIDIUM DIFFICILE INFECTION}

\section{Background/Statement of the Problem}

Despite advances in the diagnosis and treatment of Clostridium difficile infection (CDI), the prevention of CDI, particularly in the inpatient hospital setting, remains a challenge. In recent years, both the incidence and severity of (CDI) have increased, accompanied by an associated rise in mortality (Centers for Disease Control [CDC], 2015b). CDI is a serious health care associated infection and a growing health care problem. "Hospital-acquired infections (HAI) are a major concern for hospitals across the country and C. difficile is among the most dangerous," says principal investigator Leonard Mermel, D.O., Medical Director of the department of epidemiology and infection control at Rhode Island Hospital (Jefferson et al., 2013). Clostridium difficile now rivals methicillin-resistant staphylococcus aureus (MRSA) as the most common pathogen to cause hospital acquired infections (HAI) in the United States (Dubberke et al., 2014). A statistic from the 2014 update of Strategies to Prevent Clostridium Difficile Infections in Acute Care Hospital, published by the Society for Healthcare Epidemiology of America, is that patients who received ICD 9 codes for the discharge diagnosis of clostridium difficile infection more than doubled from 2000 to 2009 (Dubberke et al.). In 2011, a point prevalence survey of heath care associated infections was performed; from 183 hospitals and 10 states, 11,282 patients were randomly selected. Of those 11,282, 4.0\% had one or more HAI, and Clostridium difficile was the most commonly identified pathogen, accounting for $12 \%$ of all HAIs (Dubberke et al.). According to the CDC, an estimated half a million CDI infections were diagnosed in the United States during 2011 (CDC, 2015b). Approximately 83,000 of the patients who developed CDI experienced 
at least one recurrence and 29,000 of these patients died within 30 days of the initial diagnosis (CDC, 2015a).

The rise in CDI rates has been recognized as an issue here in Rhode Island and steadily continues to gain national recognition. Based on 2013 data published on the CDC website, Rhode Island is among ten other states whose CDI rates have exceeded the national average (CDC, 2015a). The rate of CDI in Rhode Island increased more than three fold over the past decade going from 5.21 per 1,000 discharges in 2002 to 18.87 per 1,000 in 2012, outpacing national trends and neighboring states (Jiang, et al., 2014). In the first quarter of 2013 Rhode Island ranked $1^{\text {st }}$ among the 50 states and Washington D.C. (Jiang et al.).

C. difficile infection is the major identifiable cause of antibiotic-associated diarrhea and is responsible for 15-25 percent of all cases, with a marked increase in the incidence of CDI since the turn of the 21 st century (Bartlett \& Gerding, 2008). The reemergence of CDI, coupled with an increasingly vulnerable healthcare population, has resulted in more frequent medical and surgical complications, added health care costs, and greater mortality (DePestel \& Aronoff, 2013). Hospitalized patients are considered to be especially high risk for CDI, and among inpatient cases, antibiotic treatment has been an almost universal factor in the development of CDIs (DePestel \& Aronoff). This makes hospitalized individuals who are receiving antibiotic the most susceptible. According to the CDC, more than half of all hospitalized patients will get an antibiotic at some point during their hospital stay (CDC, 2015b).

Fluoroquinolone resistance appears to have been a critical factor in the worldwide spread of the pathogen, including its persistence in the hospital environment 
(Kenneley, 2014). Due to the fact that CDI has received a lot of media and medical attention over the past several years, many hospitals have implemented strict protocols and preventative bundles to help reduce occurrence rates. Due to the nature of this resilient organism, all evidence based preventative measures should be considered. One preventative measure that is incontinently used in the prevention of CDI is oral probiotics.

Probiotic consumption is reported to exert a myriad of beneficial effects including enhanced immune response, balancing of colonic microbiota, treatment of diarrhea associated with travel and antibiotic therapy, control of rotavirus and clostridium difficile induced colitis. Probiotic bacteria attach to enterocytes and thus inhibit the binding of enteric pathogens to the intestinal mucosa by production of inhibitory substances. Emerging evidence has revealed that prevention of gastrointestinal tract colonization by a variety of pathogens such as clostridium difficile is a primary mechanism of beneficial effects mediated by probiotics. (Kaur, Chopra, \& Saini, 2001).

The American College of Gastroenterology recognizes the role of probiotics and included probiotics as a level B recommendation for the treatment of CDI (Avadhani \& Miley, 2011). It has been hypothesized that the use of probiotics, as an adjunctive therapy in patients receiving antibiotics, may provide a key intervention in reducing primary CDI (Evans \& Johnson, 2015)

Based on the compelling evidence regarding the use of oral probiotics as an adjunctive therapy it is necessary to explore if in fact providers are prescribing probiotics to patients receiving antibiotic therapy. More specifically an important question is if providers are prescribing to patients who are hospitalized and receiving Fluoroquinolone 
antibiotic. Therefore, the purpose of this research was to explore healthcare providers prescribing trends regarding Fluoroquinolone antibiotics and adjunctive probiotics use. Review of literature will be discussed next. 


\section{Literature Review}

To conduct a review of literature the following databases were searched: MEDLINE, PubMed, Ovid, CDC and World Health Organization. The terms, Clostridium difficile infection (CDI), CDI and Probiotics, prevention of CDI, methods of Prevention in CDI, what is a probiotic, physician use of probiotics, and fluoroquinolones causing CDI were used. The literature review includes the following subsections: clostridium difficile infection and contributing factors; probiotic definition and action; probiotics: usage as a preventative measure of CDI; fluoroquinolones and CDI; prescribers influence in the use of probiotics.

\section{Clostridium Difficile Infection (CDI) and Contributing Factors}

A nosocomial infection - also called "hospital acquired infection" can be defined as an infection acquired in hospital by a patient who was admitted for a reason other than that infection (World Health Organization, 2002). Clostridium difficile is a Gram-positive, spore-forming, anaerobic bacterium that when ingested can cause CDI (CDC, 2015a). Clostridium difficile infection symptoms include varying severity of diarrhea as well as abdominal pain, cramping and possible fever. Clostridium difficile is shed in feces and in order for a person to develop CDI they must be infected with a strain of C. difficile capable of making toxins in the colon. Any surface, device, or material (e.g., toilets, bathing tubs, and electronic rectal thermometers) that becomes contaminated with feces may serve as a reservoir for the Clostridium difficile spores (McDonald \& Stokowski, 2012). Clostridium difficile spores are transferred to patients mainly via the hands of healthcare personnel who have touched a contaminated surface or item. 
Clostridium difficile can live for long periods of time on surfaces (CDC, 2015a).

Additionally, unnecessary or excessive antibiotic use combined with poor infection control practices may increase the spread of $\mathrm{C}$. difficile within a facility and across facilities (McDonald \& Stokowski, 2012). Touching a patient who is colonized with CDI or interacting in the environment of a patient colonized with CDI can lead to infection (CDC, 2015a). The major difference among the three organisms is that C.difficile forms spores whereas the other infectious organisms do not. The formation of spores poses unique challenges for hand hygiene and environmental disinfection practices, since $\mathrm{C}$. difficile spores are resistant to the bactericidal effects of alcohol and the most commonly used hospital disinfectants (Dubberke et al, 2014).

As of January 2013, the Centers for Medicare \& Medicaid Services (CMS) require all acute care hospitals participating in their Inpatient Prospective Payment System to report any laboratory-identified CDI using the National Health Safety Network (NHSN). The NHSN has defined healthcare facility onset, also phrased health care facility associated CDI, as CDI symptom onset more than 3 days after admission to a healthcare facility, with day of admission being day one (Sievert et al., 2013).

\section{Probiotics: Definition and Actions}

In an expert consensus document published by the International Scientific Association for Probiotics and Prebiotics in 2013, the Food and Agriculture Organization of the United Nations and World Health Organization defined probiotics as, "live microorganisms which when administered in adequate amounts confer a health benefit on the host" (p.506). Probiotics are active living microorganisms that have a defined health 
benefit - either therapeutic or preventative when ingested in sufficient quantity (Heiberger, Hellwig, \& Ladwig, 2014). Probiotics are bacteria similar to the beneficial microbes naturally found in the human gut and are used to replace or increase an individual's microflora (Merenstein, 2012). Several mechanisms have been proposed to explain the actions of probiotics, but in most cases the exact mechanism of action is not fully understood and it is likely that a number of mechanisms are working simultaneously (Heiberger, Hellwig, \& Ladwig 2014). Probiotics protect intestinal epithelial cells by altering gut micro biota and modulating immune responses (Evans \& Johnson, 2015). Probiotic bacteria help reduce colonization of pathogenic organisms by competitively inhibiting their adhesion on the intestinal mucosa surface (Kopp-Hooliahn, 2001).

A wide variety of probiotic species are available, but the most investigated are species of $\quad$ Lactobacillus (L acidophilus, $L$ rhamnosus, L bulgaricus, $L$ reuteri, $L$ casei) and Bifidobacterium. In the United States, probiotics are available primarily as dietary supplements in capsule, tablet or powder formations. In addition, probiotics occur in yogurt, sauerkraut, and other fermented foods (Heiberger, Hellwig \& Ladwig, 2014).

Most studies evaluating probiotics in the prevention and/or treatment of C. difficile have evaluated the yeast Saccharomyces boulardii (Florastor) and the bacteria, Lactobacillus (Katz, 2005). The two most popular and studied probiotic organisms are Lactobacillus and Bifidobacterium species (Pattani, Palda, Hwang, \& Shah, 2013). An important consideration is that the effects of any bacteria are strain specific, meaning the data from research relates only to that specific strain. Research results cannot be generalized to other species or strains (Hickson, 2011). For example, L. rhamnosus GG is 
a specific bacterial strain which demonstrates a probiotic effect in the prevention of AAD (McFarland, 2006). Other strains of L. rhamnosus species may not have this effect, and likewise other species in the genus of Lactobacillus may not act as probiotics. This is because, individual strains exhibit different specific characteristics, such as resistance to gastric acid and bile, ability to colonize the mucosa, and antimicrobial activity (Jacobsen et al., 1999).

It is generally agreed that a probiotic must be capable of colonizing the intestinal tract to influence human health (Pattani et al., 2013). This requirement may disqualify many of the stains currently used in fermented dairy products. There is reasonable evidence to support the use of S. boulardii for the prevention of antibiotic-associated diarrhea at a dose of $1 \mathrm{gm}$ daily in hospitalized adults (Pattani et al.). Lactobacillus GG (LGG) is one of the most extensively studied probiotics which has proved to be beneficial in reducing the severity and duration of antibiotic associated diarrhea (Kaur, Chopra, \& Saina, 2001). When consumed in a dairy product or as a lyophilized powder, LGG colonizes the gastrointestinal track for 1-3 days in most individuals. The purported benefits for any probiotic must pass the highest standards of scientific scrutiny before the claims for its usefulness ca be accepted. In 2002 the World Health Organization (WHO) and Food and Agriculture Organization (FAO) of the United Nations issued guidelines detailing criteria that need to be fulfilled by a food product before labeling it as a probiotic (Cordina, Shaikh, Shrestha, \& Camilleri-Brennan, 2011). The guidelines also detail what information should be available on the product label. These include genus, species and strain designation, minimum viable numbers of each strain at the end of shelf-life, suggested daily intake to achieve effective dose for claimed health benefits, 
health claims (which should be backed by scientific evidence) and recommended storage conditions (Cordina, et al.).

\section{Probiotics: Usage as a Preventative Measure of CDI}

Most infection control strategies place emphasis on interruption of horizontal transmission of C. difficile between patients, their environment, and healthcare workers. Despite the fact that the use of probiotics for prevention of primary CDI is still not identified as a core CDC recommendation, further improvements in the prevention of CDI would benefit if focused on prevention of disease in those patients who are at risk (Dubberek et al.,2014). The goal of therapy when using probiotics as a preventative measure is to mitigate the effects of microbiota disruption. This approach involves the introduction of competing, nonpathogenic (probiotic) organisms into the intestinal tract to restore microbial balance (Pochapin, 2000). The theoretical premise behind this approach is that the protective intestinal microflora is damaged by antibiotic treatment; the initial antibiotic exposure thus leaves the host susceptible to colonization and subsequent infection by Clostridium difficile (Pochapin, 2000). The current CDC core recommendations for prevention include implementing an antimicrobial stewardship program, contact precautions for duration of diarrhea, hand hygiene in compliance with CDC/World Health Organization standards, cleaning and disinfecting the environment and equipment, CDI education for all staff/personnel who come in contact with patients, and laboratory based alert systems for immediate reporting of positive results (Dubberek, et al,). 
In 2011, Avadhani and Miley published a meta-analysis with the purpose to report findings from available studies that evaluate the efficacy of probiotics administered to hospitalized adults in the prevention of antibiotic associated diarrhea (AAD) and CDI. A comprehensive systematic search was conducted to identify all relevant studies on probiotic efficacy in prevention of AAD and CDI. Data synthesis was done using MAStARI software from the Joanna Briggs Institute in Australia (Avadhani \& Miley, 2011). Results revealed that administration of probiotics led to a statistically significant relative risk reduction of $71 \%$ for CDI which supports the potential role of probiotics to favorably influence the mechanisms that is responsible for antibiotic associated diarrhea and possibly CDI.

In 2013, Maziade, Pereira, \& Goldstein published an observational study in the Journal of Clinical Infectious Diseases. This study took place over a 10-year period, beginning in 2004 and ending in 2015 . In 2003 a 284- bed community hospital named Pierre-Le Gardeur (PLGH) in Quebec Canada experienced a major outbreak of CDI. They recognized that hospitals in metropolitan Montreal and the surrounding Quebec region also had a marked increase in the incidence of CDI from 5.7 cases per 1000 patient admissions in 2001 to 28.2 CDI cases per 1000 admissions in 2003. After an initial 6-month observational period that showed apparent ineffectiveness of the current standard preventative measures (SPM) alone at PLGH, they added a probiotic bundle. Beginning in January 2004 every inpatient adult on antibiotics (without any exclusion) was started on a probiotic called BioK+ (combination of Lactobacillus acidophilus CL 1285, Lactobacillus casei LBC80R and Lactobacillus rhamnosus CLR2) within 12 hours of the antibiotic prescription. Subjects were given 2 capsules per day each100 billion 
CFU (colony forming unit) and the probiotic was continued for five days' post antibiotic discontinuation. During the 10 years of observation, 44,835 inpatients received Bio $\mathrm{K}+$.

During the combined observational phase (April 2005- March 2014) data was published annually and were expressed as cases per 10,000 patient days. The average annual incidence rate of CDI at PLGH remained at values much lower than those observed in the conglomerate of other Quebec network hospitals (2.3 vs 7.5 cases per 10,000 patient- days). These values were also lower than those from equivalent hospitals (> 250 beds) (8.3 cases per 10,000 patient-days). All rate reductions observed during the initial utilization of Bio $\mathrm{K}+$ were maintained for 9 years. The CDI rate at PLGH declined from 19.0 cases per 10,000 patient days and remained at low mean levels of 2.3 cases per 10,000 patient days. This 10 - year intervention resulted in a $73 \%$ reduction of CDI cases $(\mathrm{P}<.001)$. Blood cultures were monitored a PLGH for Lactobacillus bacteremia through the 10-year study, and no Lactobacillus bacteremia were detected.

This study did reveal that it was limited because it was an observational study as opposed to a randomized control trail, and because it only studied one probiotic (Bio $\mathrm{K}+$ ). The study was sponsored by Bio K+ Plus International which may present a potential conflict of interest.

In 2013 a systematic review and meta-analysis was published by Pattani et al. to evaluate the efficacy of co-administration of probiotics with antibiotics in preventing CDI. Systematic searches of MEDLINE, Embase, and the Cochrane Central Register of Controlled Trials were searched for randomized controlled trials, published in English, of adult inpatients who were receiving antibiotics and who were randomly assigned to co- 
administration of probiotics, with or without the use of placebo. Pooled analyses revealed significant reductions in Antibiotic Associated Diarrhea (AAD) (RR 0.61, 95\% CI 0.47 to 0.79 ) and $\mathrm{CDI}$ (RR $0.37,95 \% \mathrm{CI} 0.22$ to 0.61 ) among patients randomly assigned to coadministration of probiotics. With subgroup analysis, significant reductions in rates of both AAD and CDI were retained in the subgroups of good-quality trials and the trials assessing a primarily Lactobacillus-based probiotic formulation. The interpretation made from this meta-analysis and systematic review was that probiotics used concurrently with antibiotics reduce the occurrence of AAD and CDI (Pattani et al.).

\section{Fluoroquinolones and CDI}

The CDC has identified antimicrobial use as one of the main modifiable risk factors of CDI (Chernak et al, 2005). Virtually every antibiotic has been associated with CDI although, for the past 10 years, fluoroquinolones, which were previously infrequently associated with CDI, have been found to be one of the primary precipitating antimicrobials (Dhalla \& Mamandi 2006).

Yip, Loeb, Salama, Moss, \& Olde (2001) published a case control study in the Journal of Infection Control and Hospital Epidemiology. The study took place in a 300 bed tertiary-care hospital. All adult inpatient charts were reviewed during October 1998 to December 1998 to detect individuals with a positive enzyme immunoassay for C difficile toxin. Twenty-seven patients were identified as subjects in the study. Casepatients identified with nosocomial CDI over the study period were compared with two sets of control patients. When case-patients were matched to control patients by date of admission, age, gender, and inpatient unit, the only significant risk factor in univariate 
analysis was prior use of Ciprofloxacin (odds ratio [OR], 5.5; 95\% confidence interval [CI95], 1.2-24.8; $\mathrm{P}=.03)$. Additionally, the following variables were entered into the conditional logistic regression model: renal insufficiency, use of cephalosporins, use of ciprofloxacin, and use of proton pump inhibitors. Use of ciprofloxacin was the only variable that remained significant in the final model. This study found that patients with CDI were at least five times more likely to have been exposed to ciprofloxacin than control patients (OR, 5.5 and 9.5 for the first and second sets of control patients, respectively). The authors of this study concluded that these findings raise concern about the use of quinolones in acute-care hospitals with endemic CDI.

McCusker, Harris, Perencevich, and Roghmann (2003) performed a case control study to evaluate the association between antibiotic use and CDI. The study was performed in a 778 bed Veterans Administration hospital in Maryland over a six-month period. Thirty patients who had their first occurrence of CDI were identified as subjects. All 30 patients had documentation of receiving antibiotics within six weeks prior to the positive CDI lab assay. Matched univariate analysis of risk factors for CDI showed that fluoroquinolone use odds ratio [Odds ratio] 13.5; 95\% confidence intervals $[\mathrm{CI}] 3.1$ to 58.8) and clindamycin use (OR 3.1; 95\% CI 1.0 to 9.4) were associated with developing CDI. A multivariable regression style showed that treatment with Fluoroquinolones (odds ratio 12.7, 95\%, confidence interval (CI) 2.6-61.6) was the strongest risk factor for CDI.

Sarma, Marshall, Cleeve, Tate, Oswald, and Woolfrey (2015) published 
an interrupted time-series analysis pre and post fluoroquinolone restriction for 60 months based on a Poisson distribution model. The primary aim of this study was to describe the implementation and to analyze the impact of fluoroquinolone restriction on CDI over a five-year period (2007-08 to 2011-12) in two hospitals. Approximately 93\% of cases received antibiotics within the eight weeks prior to infection and the majority received either cephalosporins (52\%) or fluoroquinolones (41\%).

Findings suggested that in June 2008, fluoroquinolone consumption decreased in half to about 5 defined daily doses (DDD) per 100 occupied bed -days (OBD). This was followed by a significant fall in CDI rate (rate ratio $0.332,95 \%$ CI: $0.240-0.460$ ) which remained low over the subsequent months. Fluoroquinolone consumption was further reduced to about $2 \mathrm{DDD} / 100 \mathrm{OBD}$ in June 2010, accompanied by further reduction in CDI rate (rate ratio: $0.394 ; 95 \% \mathrm{CI},: 0.199-0.781$ ). The researchers concluded that the reduction in fluoroquinolone usage was associated with an immediate, large, and significant reduction in CDI cases.

\section{Prescribers Influence}

Health care providers prescribe 258 million courses of antibiotics, which translates to 833 prescriptions per 1000 people, or four out of five people (Hicks \& Taylor, 2013). While antibiotic-prescribing rates declined in the 1990s, there has been a steady increase in their use since then (Ashiru-Oredope, Sharland, Charani, McNulty, \& Cooke, 2012).

To be qualified to prescribe medications, prescribers are required to undergo the necessary training to achieve a solid understanding of antibiotic pharmacotherapeutics 
(Hicks \& Taylor, 2013). Not only must the prescriber possess confidence in their prescribing methods but they must also know the risk vs. benefit of prescribing such medication. The ability to properly prescribe medication to treat disease is a major factor in the establishment of a trusting rapport with patients. Accordingly, patients must have faith in their prescriber's ability to accurately and safely prescribe medications. To remain effectively integrated in their patients' care, health care providers must consider the risk for development of CDI from antibiotic use and be familiar with common beliefs about probiotics while be prepared to discuss probiotics with patients who express an interest in pursuing these options.

One of the first studies to directly assess practicing physicians' perceptions on the efficacy, use, and practice patterns for recommending probiotics in the treatment of gastrointestinal disorders was by Williams, Ha and Ciorba (2010). The objective of this study was to determine how gastroenterologists perceive and use probiotic-based therapies in practice. A 16 item multiple choice questionnaire was developed and distributed to practicing gastroenterologists and physicians in Saint Louis, Missouri. Participants specialized in gastrointestinal disorders and were from both private community based gastroenterology practices as well as academic affiliates of two medical schools. A total of 96 invitations were sent, 56 (58\%) physicians completed the survey with a response rate of $65 \%$ among academic-based physicians and $53 \%$ of community practice physicians $(\mathrm{P}=0.30)$. Twelve responses came from continuing medical education participants including 7 primary care physicians, 1 community gastrointestinal surgeon, and 4 senior fellows. Results showed that all the private practitioners surveyed described themselves as, "somewhat familiar with the literature 
involving probiotics", and considered them safe. Additionally, 98\% $(\mathrm{n}=56)$ expressed a belief that probiotics had a role in the treatment of GI illness and symptoms (Williams et al., 2010).

Similarly, $98 \%$ of the surveyed physicians recommended probiotics for irritable bowel syndrome, Clostridium difficile-associated diarrhea and for patients during antibiotic use; because they believed that the literature supports their usage for these conditions. Of all the respondents $n=56$ both private practice physicians and academic were most familiar with the individual probiotic preparations B. infantis (Align) $89 \%$ and 93\% the commercially sold yogurt-based probiotic supplement preparations known as Danative, Yakult, and Lifeway. However, a majority of them were also familiar with Lactobacillus rhamnosus GG (Culturelle) $61 \%$ private practice physician and $42 \%$ academic medicine physician $n=56$ and Florastor (Saccharaomyces boulardii) $71 \%$ private practice physician and $39 \%$ in academic medicine physician $n=56$. Both private and academic practitioners had similarly positive responses regarding the efficacy of probiotics. Despite their tendency to prescribe the probiotics, results showed that $82 \%$ $n=56$ of the surveyed physicians reported that probiotics were "sometimes" effective, $7 \%$ $\mathrm{n}=56$ responded that they were "always" effective, and $11 \% \mathrm{n}=56$ of physicians had never recommended probiotics in their practice. In this survey, patients seen by private practitioners more commonly took probiotics for C. difficile-associated diarrhea when compared with patients of their academic counterparts, $89 \%$ versus $55 \%$. Conditions for which physicians believe literature supports probiotic usage included irritable bowel disease, antibiotics associated diarrhea, clostridium difficile, pouchitis, ulcerative colitis and crohn's (Williams et al. 2010). 
A study by Cordina, et al. (2011) aimed to investigate the current attitudes and prescribing practices of surgeons and gastroenterologists related to probiotics in the treatment of gastrointestinal disorders. A questionnaire was designed to examine the frequency of probiotic prescribing, types of probiotics used, indications for and duration of treatment and clinicians' experiences with probiotic use. A total of 220 questionnaires were mailed to consultant gastroenterologists and surgeons practicing in the United Kingdom. A total of 177 respondents, of whom 73 were surgical consultants and 104 were gastroenterologists. The response rate was therefore $73.0 \%$ for surgical consultants and $86.7 \%$ for gastroenterologists $(\mathrm{P}=0.016)$ with an overall response rate of $80.5 \%$. Of the surgical consultants who responded, 26 (35.6\%) were colorectal surgeons, $16(21.9 \%)$ upper GI surgeons, and 31 (42.5\%) were general surgeons.

Overall, 123 of the 177 respondents $(69.5 \%)$ recommended or prescribed probiotics to their patients. A total of $80.8 \%$ gastroenterologists (84 of 104) recommended or prescribed probiotics to their patients and only 53.4\% (39 of 73) of consultant surgeons did so $(\mathrm{P}=0.00013)$. When comparing the different surgical specialties, probiotics were more commonly prescribed by colorectal surgeons, of whom $88.5 \%$ ( 23 of 26 ) said they prescribed or recommended probiotics to their patients, compared to only $43.8 \%$ ( 7 of 16) of upper GI surgeons and $29.0 \%$ (9 of 31) of general surgeons (Cordina et al,2011). Of the respondents, 15.4\% indicated prescribing for the treatment or prevention of Clostridium difficile-associated diarrhea (CDAD) and 5.1\% prescribed for indication of antibiotic- associated diarrhea.

Information published specifically related to nurse practitioners (NPs) and probiotics is minimal. In the August 2015 Gastric Health edition of the Journal of Nurse 
Practitioner Avadhani and Steefel (2012) published a review that sought to educate nurse practitioners on the use and benefits of probiotics. This article reinforced the accountability that nurse practitioners share along with their physician's colleagues in their role regarding the use of probiotics. Amidst increasing information of probiotics, understanding the use and purpose of probiotics is still a source of confusion for the average consumer. Probiotics are readily available over the counter (OTC) and can be purchased in capsule, tablet, powder forms, or in yogurt. Advertisers promote the advantages of probiotics without a full explanation of the product. Nurse Practitioners should be educated the basics of probiotics, criteria for patient use, considerations regarding affordability of the medication and whether use of a probiotic will fit into the patient's lifestyle. Avadhani and Steefel, 2012 also emphasized the importance of NPs having knowledge of various strains of probiotics, especially the most common strains, as the benefits of one strain may not hold true to other strains and the suitability of probiotics for their patient may be impacted by the various strains. Knowledge of these factors is important for an NP's prescriptive advice.

The theoretical framework which guided this study will be discussed next. 


\section{Theoretical Framework}

The American Association of Colleges of Nursing (AACN) Synergy Model was chosen to guide the framework of this research. The AACN Synergy Model for Patient Care was developed to link clinical practice with patient outcomes. This model incorporates what the patient expects, what the health care professional should provide, and what the health care system should deliver. Additionally, the framework also supports that optimal outcomes result from the synergy of nurses' competencies and the nurses' ability to meet the needs of patients, their families, and the system (Kaplow \& Hardin, 2004).

This model incorporates patient characteristics including, predictability, and complexity, risk of death, vulnerability, and participation in care. While conducting this research these patient characteristics helped to guide and direct the focus and intentions of the study. For instance, it is reasonable to assume, that the majority of hospitalized patients expect that to receive optimal care and be healed as opposed to gaining additional medical problems while being cared for in the hospital. This concept aligns with the synergy model's components of patient expectations.

Vulnerability, is the patient's level of susceptibility to actual or potential stressors that may or may not adversely affect the patient outcomes (Kaplow \& Hardin, 2004). As stated earlier the CDC (2015 a) projects that more than half the patients in a hospital are receiving antibiotics which translates to a large population who are vulnerable. Predictability, allows us to expect or anticipate a certain course of events. 
The Synergy Model also incorporates nurse characteristics including clinical judgment, advocacy, collaboration, and system thinking (Kaplow \& Hardin, 2004). These characteristics were particularly pertinent to this research study. All of these characteristics embody the responsibility and impact that nurses and/or providers have in utilizing all of the existing preventative measures for the development of CDI. Clinical judgment speaks to the health care professional's ability to recognize individuals who are at risk for $\mathrm{CDI}$ as well as their knowledge base as to what makes a risk factor a risk factor. Additionally, collaboration and system thinking are two vital components of this model that pertained to the core themes of this research study (Kaplow \& Hardin, 2004). Prevention of CDI in many ways betters the system within the hospital as well as promotes health outside the hospital doors. For that reason, CDI acquired infections are tracked by the individual inpatient unit and hospital, but is also tracked by each state department of health and extends all the way to the CDC for national tracking. As a result, CDI prevention is the responsibility of more than just the nurse or the provider, but rather it's the responsibility of the whole system.

The Synergy Model that can also guide nursing research and care across multiple clinical populations (Kaplow \& Hardin, 2004). The Synergy Model has guided this study by utilizing the concept of meeting patient needs and influencing patient outcomes. With this model as a guide, the research results have helped to provide insight into how nurses and licensed independent practitioners can advocate for their patients by considering the additional preventative measures of probiotic in prevention of hospital acquired CDI.

The methods guiding this study will be presented in the next section. 


\section{Method}

\section{Purpose}

The purpose of this research was to explore healthcare providers prescribing trends regarding Fluoroquinolone antibiotics and adjunctive probiotics in patients with hospital acquired CDI.

\section{Design}

This study was a descriptive retrospective chart review of patients admitted from the time frame of March 29, 2015 to January 31, 2016. Patient records with a discharge diagnosis of hospital acquired CDI during that time period were reviewed.

\section{Sample and Site}

Inclusion criteria included records of both male and female individuals 18 years of age and older who received care as inpatients at The Miriam Hospital, a 247- bed acute care, university affiliated teaching hospital located in Providence, Rhode Island. All participants needed to have been diagnosed with hospital acquired clostridium difficile via ICD 9 and 10 (International Classification of Disease) during their hospital stay at the study site. Exclusion criteria included records of patients who were admitted with a previous diagnosis of CDI or were admitted to rule out a community acquired CDI.

\section{Procedure}

Permission was obtained from the Chief Nursing Officer of TMH as well as the director of Infection Control at TMH. The Lifespan and RIC IRBs reviewed and approved this study. Data for this research were obtained through a retrospective chart review conducted at The Miriam Hospital (TMH). Participants with hospital acquired CDI were identified under the guidance of the institution's Department of Infection Control through a tracking system called Theradoc. The researcher was provided access 
to electronic medical records upon IRB approval. Data for the specific participants who were diagnosed with hospital acquired CDI between the dates of March 29, 2015 to January 31st, 2016 were provided to the researcher by the Director of TMH Infection Control department. The researcher then categorized charts based upon whether or not the participants received an oral or intravenous Fluoroquinolone antibiotic and/or if they were receiving a probiotic. Only data meeting the inclusion criteria and required for analysis were extracted and no identifying information was collected.

\section{Measurement}

A worksheet (Appendix A) was developed by this researcher and was used to gather the above information. The data collection tool was developed based on the review of the literature, the identified variables of interest, and clinical experience.

\section{Data Storage and Analysis}

Data were stored on a Lifespan encrypted and approved USB zip drive and kept with the researcher in a locked safe. After completion of the research, the USB zip drive was locked in the primary investigator's office.

Basic descriptive statistics was utilized to analyze and summarize the probiotic prescribing trends of health care providers at this institution during the specified time frame. Additionally, comparisons were made between the percentages of subjects who developed CDI that were also on a fluoroquinolone antibiotic and those that were on a prophylactic probiotic.

Next, study results will be presented. 


\section{Results}

A total of 96 records were reviewed; of those records, 16 were excluded for not meeting inclusion criteria. The remaining 80 records met inclusion criteria $(n=80)$ because subjects were given a diagnosis of hospital acquired clostridium difficile infection between the dates of March 29, 2015 and January 31, 2016. Table 1 below illustrates ....

Table 1 Core Data

\begin{tabular}{|l|l|}
\hline Total number of hospital acquired (HA) CDI patients & $\mathbf{n = 8 0}$ \\
\hline Patients receiving fluoroquinolone when diagnosed with CDI & $\mathbf{n = 4 1 ~ ( 5 3 \% )}$ \\
\hline Of the total CDI patients (80) those also receiving probiotics & $\mathbf{n = 2 2 ~ ( 2 8 \% )}$ \\
\hline \% of Fluoroquinolone receiving patients (41) also prescribed probiotic & $\mathbf{5 5 \%}$ \\
\hline
\end{tabular}

Of those 80 subjects, $53 \%(n=41)$ were receiving a Fluoroquinolone antibiotic when diagnosed with CDI and $22(\mathrm{n}=28 \%)$ were prescribed a probiotic. Fifty-five percent of the subjects who were prescribed a Fluoroquinolone $(n=41)$ were also prescribed a probiotic. The most commonly prescribed Fluoroquinolone was Ciprofloxacin (76\%). A further breakdown of the types of Fluoroquinolones prescribed is illustrated in Table 2. 
Table 2

Types of fluoroquinolones prescribed $\quad N=41$

\begin{tabular}{|c|cc|}
\hline Type of Fluoroquinolone & \# and \% from the total who received a Fluoroquinolone $(\mathrm{n}=41)$ \\
\hline Ciprofloxacin & $31 / 41 \quad \mathbf{( 7 6 \% )}$ \\
\hline Levaquin & $8 / 41 \quad \mathbf{( 2 0 \% )}$ \\
\hline Moxifloxacin & $2 / 41 \quad \mathbf{( 5 \% )}$ \\
\hline
\end{tabular}

Florastor, an oral tablet which is also labeled as Saccharomyces boulardii was the most commonly prescribed probiotic; accounting for $68 \%$ of the probiotics prescribed $(n=22)$. The two additional probiotics were shown to be less commonly prescribed. Table 3 illustrates the prescribed probiotics.

Table 3

Types of probiotics prescribed $\quad \boldsymbol{n}=\mathbf{2 2}$

\begin{tabular}{|c|c|}
\hline Type of Probiotic prescribed & \# and \% that were prescribed $(\mathrm{n}=22)$ \\
\hline Florastor 250mg two times daily & $15 / 22 \quad \mathbf{( 6 8 \% )}$ \\
\hline Lactinex 1 package two times daily & $4 / 22 \quad(\mathbf{1 8 \%})$ \\
\hline Culturelle (Acidophilus) 1-2 tablets daily & $3 / 22 \quad(\mathbf{1 4 \%})$ \\
\hline
\end{tabular}


Of the 80 subjects, $28 \%(n=22)$ were prescribed a probiotic at some point in their hospital stay. In the institution studied, physicians constitute the majority of practitioners and the number of physician assistants (PAs) and nurse practitioners (NPs) are about equal. A review of prescribing trends specific to each credentialed prescriber is illustrated in Table 4.

Table 4

Prescribers who prescribed probiotic $\quad n=22$

\begin{tabular}{|c|c|}
\hline Prescriber Credentials & \# and \% of probiotics prescribed $(\mathrm{n}=22)$ \\
\hline Physician & $12 / 22 \quad \mathbf{( 5 4 \% )}$ \\
\hline Nurse Practitioner & $7 / 22 \quad \mathbf{( 3 2 \% )}$ \\
\hline Physician Assistant & $3 / 22 \quad \mathbf{( 1 4 \% )}$ \\
\hline
\end{tabular}

Of the 22 subjects prescribed a probiotic, $18 \%$ were prescribed prior to the diagnosis of CDI. Thirty-six percent of the probiotics $(n=22)$ were prescribed at the time of CDI diagnosis. A significant percentage (23\%) of probiotics were prescribed one day after CDI diagnosis; this may have been due to late diagnosis. A large percentage of patients $(81 \%)$ that were receiving probiotics while hospitalized did not continue probiotic at time of discharge; this raises curiosity. What are the reasons for discontinuing therapy and is this due to a lack of provider knowledge or confidence in prescribing probiotic therapy? Table 5 illustrates timing of probiotics being initiated. 
Table 5

Initiation of Probiotic Therapy $\quad n=22$

\begin{tabular}{|l|c|}
\hline Time probiotics were initiated & \# and \% initiated at that time \\
\hline Probiotic prescribed before CDI diagnosis & $4 / 22 \quad \mathbf{( 1 8 \% )}$ \\
\hline Probiotic prescribed at time of CDI diagnosis & $8 / 22 \quad(\mathbf{3 6 \%})$ \\
\hline Probiotic prescribed one day after CDI diagnosis & $5 / 22 \quad(\mathbf{2 3 \%})$ \\
\hline Probiotic prescribed at time of discharge & $5 / 22 \quad(\mathbf{2 3 \%})$ \\
\hline
\end{tabular}

Summary and conclusions will be presented next. 


\section{Summary and Conclusions}

Nosocomial infections contribute as major causes of death and increased morbidity among hospitalized patients and represent a significant burden to both the patient and for public health. The advancing age of patients admitted to the hospital, the greater prevalence of chronic diseases, the increased use of diagnostic procedures and therapeutic medications all affect the host defenses and will contribute to the continuing incidence of nosocomial infections in the future (World Health Organization, 2002). There are a variety of factors which place a patient at risk for the development of hospital acquired CDI, including decreased immunity, the increasing variety of medical procedures and invasive techniques creating potential routes of infection, and the transmission of drug-resistant bacteria among crowded hospital populations, where poor infection control practices may facilitate transmission (Sehulster, 2010).

Clostridium difficile now rivals methicillin-resistant staphylococcus aureus (MRSA) as the most common pathogen to cause hospital acquired infections (HAI) in the United States (Dubberke et al., 2014). Strategies to Prevent Clostridium Difficile Infections in Acute Care Hospital, published by the Society for Healthcare Epidemiology of America (2014) reported that patients who received ICD 9 codes for the discharge diagnosis of clostridium difficile infection more than doubled from 2000 to 2009 (Dubberke et al.). An estimated half a million CDIs were reported in the United States in 2011 (CDC, 2015a). Approximately 83,000 of those patients experienced at least one recurrence and 29,000 of them died within 30 days of the initial diagnosis (CDC, 2015a). In a study by Williams et al., in 2010, 98\% ( $\mathrm{n}=56)$ of gastroenterologists expressed a 
belief that probiotics had a role in the treatment of GI illness and symptoms including CDI.

A notable inconsistency in the prescribing of probiotics as an adjunctive therapy to prevent hospital acquired CDI inspired the purpose of this research. A retrospective chart review examined the prescribing trends for probiotics in patients hospitalized at The Miriam Hospital in Providence Rhode Island from March 29, 2015- January 31st 2016. The sample included records of patients who had been diagnosed with hospital acquired CDI during that timeframe $(n=80)$. Descriptive statistics were performed; $53 \%(n=41)$ were receiving a Fluoroquinolone at the time CDI was diagnosed. Florastor (Saccharomyces boulardii) was identified as the most commonly prescribed probiotic, making up $68 \%$ of the probiotics prescribed to this population, with the second most common being Lactinex (18\%). Further analysis of the data was unable to identify a strong association between CDI diagnosis and lack of early probiotic prescribing prior to diagnosis. Of the 80 patients who developed hospital acquired CDI, only $18 \%(n=22)$ received probiotics early enough that it would be considered a preventative approach, yet those $18 \%$ still acquired CDI.

Of the 80 patients diagnosed with hospital acquired CDI, over half (53\%) were receiving a Fluoroquinolone at the time of diagnosis, suggesting a potential association between Fluoroquinolone use and the acquiring of CDI. Twenty-eight percent $(n=)$ of the patients who developed hospital acquired CDI were receiving a probiotic and of those prescribed a probiotic $(n=22)$, the majority were initiated at time that CDI was diagnosed. Probiotics protect intestinal epithelial cells by altering gut micro biota and modulating immune responses, reduce colonization of pathogenic organisms by 
inhibiting their adhesion to the in intestinal mucosa surface. Therefore, prescribing a probiotic at time of CDI diagnosis is understandable yet it does not align with using probiotics as a preventative measure. In order for probiotics to serve as an adjunctive intervention in primary prevention of CDI, they must be prescribed prior to acquiring CDI (Evans \& Johnson, 2015). In this study, $18 \%(n=22)$ were prescribed prior to CDI diagnosis and that the majority of probiotics $(46 \% ; n=22)$ were prescribed after CDI diagnosis, with all of those subjects still developed CDI.

The development of CDI is multifactorial in that many variables s can contribute to its development. Some of those factors include, but are not limited to, poor infection control measures, immunocompromised health state, alerted or impaired gastrointestinal health, the age of the patient (very young and very old at greater risk), long term/chronic antibiotic, proton pump inhibitor or chemotherapy use (World Health Organization, 2002). It cannot be concluded that the development of CDI is solely due to a lack of probiotic use.

The limitations in this study included that the researcher did not consider certain subject characteristics that could be key factors in the development of CDI, including the state of health, age, past medical history and institutional factors (such as cleanliness, outbreaks, or infection control measures). Also, the inclusion criteria included only the comparison of probiotics to Fluoroquinolones and did not compare any other class of antibiotics. The study was also limited by the relatively small sample size and the retrospective nature of the study. The results of this study illustrated that prescribing probiotics at the initiation of antibiotic therapy was not a common practice among physicians, nurse practitioners, and physician assistants at this institution. Only $28 \%$ of 
patients $(n=80)$ were prescribed a probiotic during their hospitalization. Overall prescribing patterns around the use of probiotics are inconsistent. Nurse practitioner prescribers were for the most part equal contributors in the prescribing of probiotics.

Recommendations and implications for advanced practice will be presented next. 


\section{Recommendations and Implications for Advanced Nursing Practice}

As the field of probiotics continues to expand in the United States, so does the need for practitioners to be aware of probiotic use. Amidst increasing information about probiotics, prescribers' and consumers' understanding of use and indications remains a source of confusion. Probiotics are readily available over the counter (OTC) and can be purchased in capsule, tablet, powder, and yogurt. Advertisers promote the advantages of probiotics without a full explanation of the product. Advanced practice nurses should be educated on the basics of probiotics as well as their indications for use. Advanced practice nurses should also be familiar with the various strains of probiotics, the most common strains, and that the benefits of one strain may not hold true to other strains. Knowing this is important for an advanced practice nurses' prescriptive advice. Based on the results of the above study, nurse practitioners were for the most part equal contributors in the prescribing of probiotics. This is encouraging, yet, the fact that such a large percentage of patients that were not continued on their probiotic at time of discharge raises curiosity. What are the reasons for discontinuing therapy and is this due to a lack of knowledge or confidence in probiotic therapy? A recommendation for institutions is to consider incorporating annual education for all clinicians regarding the detrimental downstream effects of overprescribing antibiotic and under prescribing probiotics.

To aid in providing the best patient care, advanced practice nurses should be cognizant of the fact that hospital-acquired infections such a CDI can lead to functional disability, emotional stress, and disabling conditions that reduce a patient's quality of life. Additionally, the economic costs attached to such infections is demanding to an 
institution. Hospital acquired infections such as CDI can increase patient length of stay, limit hospital reimbursement, and injures the hospital's infection rate. All of these factors can negatively impact a hospital's ability to receive/renew licensure, accreditations and meeting certain standards for delivery of care and safety. As advanced practice nurses who are incorporating system measures into their practice, hospital acquired infections such as CDI can carry a magnitude of implications. Considering adjunctive therapy such as probiotics that can help reduce the risk of CDI is a responsibility each practitioner holds with their license to practice.

The lack of strong evidence-based research studies to support the use of probiotics in CDI prevention is a contributing factor to its failed adoption into practice. Policy change often stems from large, well-known, published studies sponsored by pharmaceutical companies and /or institutions that gain acknowledgment and consideration by the medical community. Although studies supporting the incorporation of probiotic therapy in the prevention of CDI remains scarce, the evidence that does exists has potential for expanded use. Momentum has been made but lack of firm scientific evidence truly supports the need for further research. Such research could be a collaborative effort amongst multiple disciplinarians such as advanced practice nurses, pharmacy and infection control. This multi-disciplinary approach could maximize the study's potential and influence policy change.

Institutions should consider adding probiotic therapy to CDI prevention bundles. Include probiotic therapy along with the already implemented CDI prevention tools; such as hand hygiene, staff education, contact precautions, disinfection of spores. The institution in which the data for this study was collected, does not currently support the 
use of probiotic therapy in the prevention of CDI. It would be recommended that this institution consider adding probiotic therapy to their already existing CDI prevention bundle and collect data to see if it's incorporation produces a reduction in hospital acquired CDI rates. As suggested above, collaborative efforts among multidisciplinarians such as advanced practice nurses, pharmacy and infection control would make the most impact. This multi-disciplinary group should work with the institution's providers to identify and explore the barriers to the use of probiotics. 


\section{References}

Ashiru-Oredope, D., Sharland, M., Charani, E., McNulty, C., \& Cooke, J. (2012). Improving the quality of antibiotic prescribing in the NHS by developing a new antimicrobial stewardship program: "Start Smart--Then Focus". Journal of Antimicrobial Chemotherapy, 1, 151-163. doi: 10.1093/jac/dks202

Avadhani, A. \& Steefel, L. (2012). Probiotics: A review for NPs. The Nurse Practitioner, 40 (8), 50-54. doi: 10.1097/01.npr.0000469256.36238.7f

Avadhani, A. \& Miley, H. (2011). Probiotics for prevention of antibiotic-associated diarrhea and Clostridium difficile -associated disease in hospitalized adults - A meta- analysis. Journal of American Academy of Nurse Practitioners, 23, 269274.

Bartlett, J., \& Gerding, D. (2008). Clinical recognition and diagnosis of clostridium difficile infection. Journal of Clinical Infectious Disease, 46, 12-18. Retrieved from http://cid.oxfordjournals.org/content/46/Supplement_1/S12.full.pdf

Center for Disease Control and Prevention. (2015a). Hospital associated infections. Retrieved from http://www.cdc.gov/HAI/organisms/cdiff/Cdiff_clinicians.html

Center for Disease Control and Prevention. (2015b). Nearly half a million Americans suffered from clostridium difficile infections in a single year. Retrieved from http://www.cdc.gov/media/releases/2015/p0225-clostridium-difficile.html 
Chernak, E., Johnson, C., Weltman, A., McDonald, L., Wiggs, L., Killgore, G, . . Lewis, E. (2005). Severe clostridium difficile-associated disease in populations previously at low risk-four states. Morbidity and Mortal Weekly Report, 54(47), 1201-1205.

Cordina, C., Shaikh, I., Shrestha, S., \& Camilleri-Brennan, J. (2011). Probiotics in the management of gastrointestinal disease: Analysis of the attitudes and prescribing practices of gastroenterologists and surgeons. Journal of Digestive Diseases, (12)6, 489-496. doi: 10.1111/j.1751-2980.2011.00534.x

DePestel, D. \& Aronoff, D. (2013). Epidemiology of Clostridium Difficile Infection. Journal of Pharmacy Practice, 26(5), 464-475. doi: 10.1177/0897190013499521

Dhalla, I., Mamandi, M., Simor, A., Kopp, A., Rochon, P., \& Iuurlink, D., (2006). Are broad spectrum fluoroquinolones more likely to cause clostridium difficile associated diseases? American Society of Microbiology, 50(9), 3216-3219.

Dubberke, E., Carling, P., Carrico, R., Loo, V., Donskey, C., . . Gerding, D. (2014). Strategies to prevent clostridium difficile infection in acute care hospitals: 2014 Update. Infection Control and Hospital Epidemiology. Retrieved from http://journals.cambridge.org/action/displayAbstract?fromPage=online $\&$ aid=9496 $\underline{695 \& \text { fulltextType }=\text { RA\&fileId }=\text { S0195941700093279 }}$ 
Evans, C. \& Johnson, S. (2015). Prevention of clostridium difficile infection with probiotics. Clinical Infectious Disease, 60(2), 122-128. doi: 10.1093/cid/civ138

Heiberger, A., Hellwig, T., \& Ladwig, J. (2014). The role probiotics in children with gastrointestinal disease. U.S Pharmacists, 39(5), 22-26.

Hicks, L. \& Taylor, T. (2013). U.S. outpatient antibiotic prescribing, 2010. New England Journal of Medicine, 368, 1461-1462. doi: 10.1056/NEJMc1212055

Hickson, M. (2011). Probiotics in the prevention of antibiotic-associated diarrhea and clostridium difficile infection. Therapy of Advanced Gastroenterology, 4(3), 185197. doi: $10.1177 / 1756283 \times 11399115$

Jacobsen, C., Rosenfeldt, N., Hayford, A., Moller, L., Michaelsen, K., Paerregaard A., \& Jakobsen, M. (1999). Screening of probiotic activities of forty-seven strains of Lactobacillus spp. by in vitro techniques and evaluation of the colonization ability of five selected strains in humans. Application of Environmental Microbiology, 65(11), 4949-4956 
Jefferson, J., Blanchard, K., Parenteau, S., Mathis, B., Chapin, K., \& Machan, J. (2013). Rhode Island Hospital reduces incidence of hospital-associated c. difficile by 70 percent. Retrieved from http://www.rhodeislandhospital.org/Newsroom/News.aspx?NewsId=57538/Rhod e-Island-Hospital-Reduces-Incidence-of-Hospital-Associated-C-difficile-by-70Percent/

Jiang, Y., Baier, R., Morphis, B., Mermel, L., \& Viner-Brown, S. (2014). Rhode Island cdiff infection trends and laboratory ID events ranking. Rhode Island Medical Journal, 60-63. Retrieved from http://rimed.org/rimedicaljournal/2014/06/201406-60-health-cdiff.pdf

Kaplow, R. \& Hardin, S. (2004). Synergy for Clinical Excellence: The AACN Synergy Model for Patient Care. Retrieved from https://books.google.com/books?hl=en\&lr=\&id=qSUv02N3z6YC\&oi=fnd\&pg=P $\underline{\mathrm{R} 11 \& \mathrm{dq}=\mathrm{aacn}+\mathrm{synerg} y+\operatorname{model} \& o t s=\mathrm{RZF} \_1 \mathrm{XsQST} \& \operatorname{sig}=\mathrm{vsVSnJwGQwn} 23 \mathrm{id} 23 \mathrm{i}}$

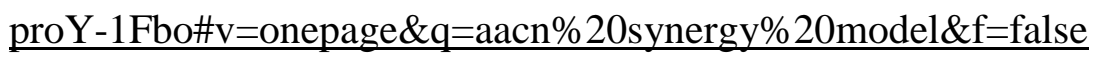

Katz, J. (2006). Probiotics for the prevention of antibiotic-associated diarrhea and Clostridium difficile diarrhea. Journal of Clinical Gastroenterology, 40(3), 249255. 
Kaur, I., Chopra, K., \& Saini, A. (2001). Probiotics: potential pharmaceutical applications. European Journal of Pharmaceutical Sciences, 15(1), 1-9.

Kenneley, I. (2014). Clostridium difficile infection is on the rise. American Journal of Nursing, 114(3), 62-67. doi: 10.1097/01.naj.0000444501.54723.5b

Kopp-Hoolihan, L., (2001). Prophylactic and therapeutic uses of probiotics: a review. Journal of American Dietary Association, 101(2), 229-239.

Maziade, P., Pereira, P., \& Goldstein, E. (2015). A decade of experience in primary prevention of clostridium difficile infection at community hospital using the probiotic combination lactobacillus acidophilus CL1285, lactobacillus casei LBC8oR, and lactobacillus rhamnosus CLR2 (Bio K+). Clinical Infectious Disease, 60(2), 144-147. doi: 10. 1093/cid/civ178.

McCusker, M., Harris A., Perencevich, E., \& Roghmann, M. (2003). Fluoroquinolone use and clostridium difficile-associated diarrhea. Emerging Infectious Diseases Journal. Retrieved from: http://wwwnc.cdc.gov/eid/article/9/6/02-0385

McDonald, C. \& Stokowski, L. (2012). Refining our approach to clostridium difficile prevention. Medscape, 2012 March 21, Retrieved from http://www.medscape.com/viewarticle/760505_2 
McFarland, L. (2006). Meta-analysis of probiotics for the prevention of antibiotic associated diarrhea and the treatment of Clostridium difficile disease. American Journal of Gastroenterology, 101, 812-822.

Merenstein, S. (2012). Probiotics for GI health in 2012: Primary issues and updates. Retrieved from http://www.primaryissues.org/2012/11/probiotics_pi161/

Pattani, R., Palda, V., Hwang, S., \& Shah, P. (2013). Probiotics for the prevention of antibiotic-associated diarrhea and clostridium difficile infection among hospitalized patients: systematic review and meta-analysis. Open Medicine, 7 (2), $56-67$.

Pochapin, M. (2000). The effects of probiotics on clostridium difficile diarrhea. American Journal of Gastroenterology, 95 (1), S11-3.

Sarma, J., Cleeve, M., Tate, D., Oswald., T., \& Woofrey, S. (2015). Effects of fluoroquinolone restriction (from 2007 to 2012) on clostridium difficile infections: interrupted time-series analysis. Journal of Hospital Infection, 91(1), 68-73. doi: 10.1016/j.jhin.2015.05.006. 
Sehulster, C. (2010). Clostridium difficile excerpt: Guideline for environmental infection control in health-care facilities, 2003. Retrieved from http://www.cdc.gov/HAI/organisms/cdiff/Cdiff_excerpt.html

Sievert, D., Ricks, P., Edwards, J., Schneider, A., Patel, J., Srinivasan, A., ... National Healthcare Safety Network (NHSN) Team and Participating NHSN Facilities (2013). Antimicrobial-resistant pathogens associated with healthcareassociated infections: summary of data reported to the National Healthcare Safety Network at the Centers for Disease Control and Prevention, 2009-2010. Infection Control Hospital Epidemiology, 34(1), 1-14. doi: 10.1086/668770

Williams, M., Ha, C., \& Ciorba, M. (2010). Probiotics as therapy in gastroenterology. Journal of Clinical Gastroenterology, 44 (9), 631-636. doi:

10.1097/mcg.0b013e3181d47f5b

World Health Organization (2002). Prevention of hospital - acquired infections: A practical guide. Retrieved from: http://apps.who.int/medicinedocs/documents/s16355e/s16355e.pdf

Yip, C., Loeb, M., Salama, S., Moss, L., \& Olde, J. (2001). Quinolone use as a risk factor for nosocomial clostridium difficile-associated diarrhea. Infection Control and Hospital Epidemiology, 22 (9), 572-575. 


\section{Appendix A}

\begin{tabular}{|c|c|c|c|c|c|c|c|c|c|}
\hline \multirow[t]{2}{*}{ DATA POINTS } & \multicolumn{9}{|c|}{ REVIEW OF PATIENT RECORD } \\
\hline & 1 & 2 & 3 & 4 & 5 & 6 & 7 & 8 & 9 \\
\hline $\begin{array}{l}\text { Day of hospitalization tha } \\
\text { CDI was acquired }\end{array}$ & & & & & & & & & \\
\hline $\begin{array}{l}\text { Was a Fluroquinolone } \\
\text { prescribed? }\end{array}$ & & & & & & & & & \\
\hline $\begin{array}{l}\text { If yes, which Fluroquinold } \\
\text { was prescribed? }\end{array}$ & & & & & & & & & \\
\hline $\begin{array}{l}\text { Hospital day that } \\
\text { Fluroquinolone was } \\
\text { prescribed. }\end{array}$ & & & & & & & & & \\
\hline Was a probiotic prescribe & & & & & & & & & \\
\hline $\begin{array}{l}\text { Hospital day that probioti } \\
\text { was initiated? }\end{array}$ & & & & & & & & & \\
\hline $\begin{array}{l}\text { Which probiotic was } \\
\text { ordered? }\end{array}$ & & & & & & & & & \\
\hline $\begin{array}{l}\text { Probiotic was ordered by } \\
\text { APRN, PA, or MD }\end{array}$ & & & & & & & & & \\
\hline
\end{tabular}

